\title{
Structural Properties of $\mathrm{SrTiO}_{3} / \mathrm{GaAs}$ Hetero-interfaces
}

\author{
Liang Hong ${ }^{1}$, Ravi Droopad ${ }^{2}$, Serdar Öğ̈̈t ${ }^{1}$ and Robert F. Klie ${ }^{1}$ \\ 1. Department of Physics, University of Illinois at Chicago, Chicago, IL \\ 2. Ingram School of Engineering, Texas State University, San Marcos, TX
}

The $\mathrm{SrTiO}_{3} / \mathrm{GaAs}$ hetero-interface is being studied due to the interest of using it in metal-oxidesemiconductor field-effect transistors, where the GaAs substrate would act as the semi-insulating base material and the $\mathrm{SrTiO}_{3}$ would act as the barrier oxide layer between the GaAs and the gate material. Previously, $\mathrm{SrTiO}_{3}$ ultra-thin film deposited on As-terminated GaAs substrate have been studied experimentally [1-3]. SrO termination for $\mathrm{SrTiO}_{3}$ is found to be more stable than $\mathrm{TiO}_{2}$ at the interface. However, whether Ga- or As-terminated GaAs is more stable at the interface remains unclear. In this work, GaAs thin film layers are grown on $\mathrm{SrTiO}_{3}$ instead of using $\mathrm{GaAs}$ as substrate. The $\mathrm{SrTiO}_{3} / \mathrm{GaAs}$ hetero-interface is characterized using atomic-resolution Z-contrast imaging and energy dispersive spectroscopy (EDS) mapping, along with density functional theory (DFT) calculations.

The sample used in this work is grown using molecular beam epitaxy method. A $10 \mathrm{~nm}$ Sr-terminated $\mathrm{SrTiO}_{3}$ thin film is grown on $\mathrm{Si}(001)$ substrate with a $4^{\circ}$ miscut in the [110] direction and a $2 \mathrm{~nm}$ thick $\mathrm{SiO}_{2}$ buffer layer. Simultaneous $\mathrm{Ga}$ and $\mathrm{As}_{2}$ are exposed to the $\mathrm{SrTiO}_{3}$ surface to form a $1 \mu \mathrm{m}$ thick epitaxial GaAs layer. The Z-contrast images (Figure 1a and b) are obtained using the aberrationcorrected JEOL JEM-ARM200CF scanning transmission electron microscopy (STEM) at 200kV. Figure 1a shows the layered structure of the sample. Step structures are observed at $\mathrm{SrTiO}_{3}(001)$ surface. Figure $1 \mathrm{~b}$ shows the atomic structure at the $\mathrm{SrTiO}_{3} / \mathrm{GaAs}$ interface, and can be separated into two areas (Area I and Area II) according to the two steps at $\mathrm{SrTiO}_{3}$ surface. In Area I, we find that the $\mathrm{SrTiO}_{3}[100]$ direction is parallel to GaAs[110], and the heterointerface appears sharp with the GaAs dumbbells in perfect registry with the $\mathrm{O}$ atoms in the $\mathrm{SrO}$ terminating layer. Due to the step of one unit cell of $\mathrm{SrTiO}_{3}$ $(3.90 \AA), 3 / 4$ unit cell of GaAs (4.24 $\AA$ ) is missing at the interface in Area II. Therefore, GaAs columns in Area II are not perfectly aligned with $\mathrm{SrTiO}_{3}$ columns due to the lattice mismatch as shown in the image. The sequence of the GaAs dumbbell is determined using the intensity line scan in the Z-contrast image along with atomic-resolution EDS mapping as shown in Figure $2 \mathrm{a}$ and $\mathrm{b}$. The intensity profile is consistent with the EDS mapping that the sequence of the GaAs dumbbell in the Z-contrast image is GaAs, which means, at the interface, the GaAs film is terminated with Ga in Area I while terminated with As in Area II as the structural model insets shown in Figure 1b. According to our DFT calculations [4], the $\mathrm{SrO} / \mathrm{Ga}$ interface with $\mathrm{Ga}-\mathrm{O}$ bonding is energetically most favorable among all the proposed configurations, which is in good agreement with the Z-contrast image in Area I. The interfacial structure becomes more interesting when $\mathrm{SrTiO}_{3}$ steps are introduced as shown in Area II. A more detailed study of the step structures using electron energy-loss spectroscopy will be presented where the fine structure of interfacial atoms will be examined. [5]

References:

[1] R. F. Klie et al, Appl. Phys. Lett. 87 (2005) 143106.

[2] Q. Qiao et al, Phys. Rev. B. 85 (2012) 165406.

[3] Y. Liang et al, Appl. Phys. Lett. 85 (2004) 1217.

[4] L. Hong et al, Microscopy and Microanalysis 21 S3 (2015) 1647.

[5] This work was supported by the National Science Foundation (Grant No. DMR-1408427). 
(a)

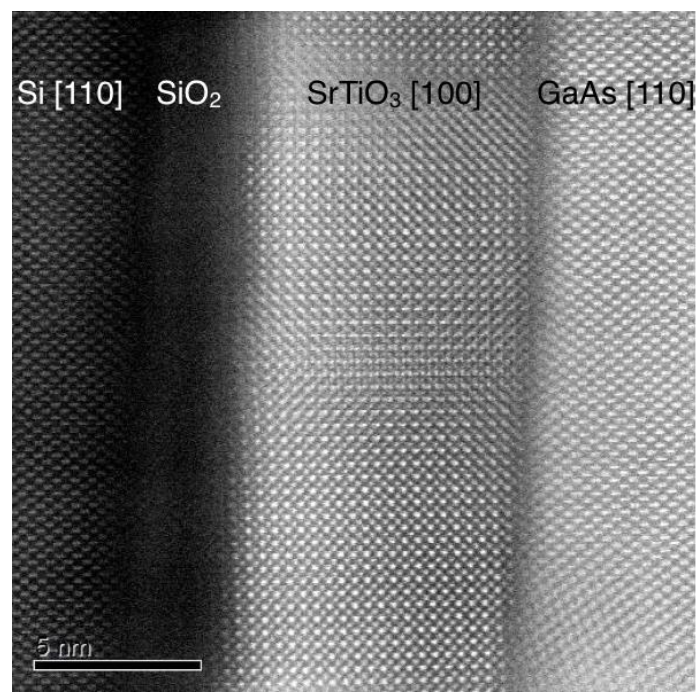

(b)

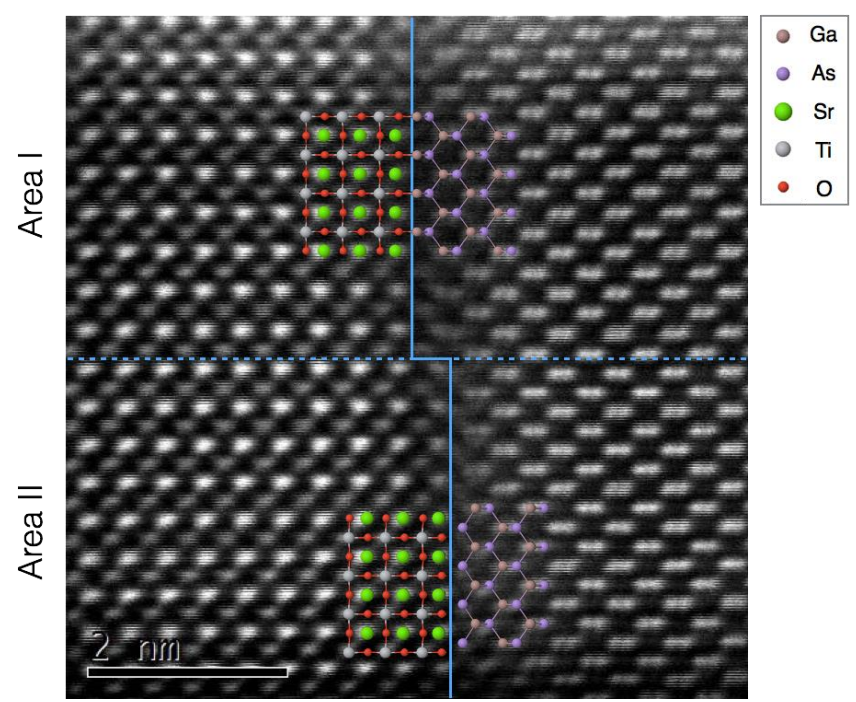

Figure 1 (a) Cross-sectional Z-contrast STEM image of the layered structure of the sample used in this work. (b) (Color online) Atomic-resolution Z-contrast STEM image of the $\mathrm{SrTiO}_{3} / \mathrm{GaAs}$ heterointerface. The proposed structural models of the atomic arrangement at the interface are shown as insets. The step structure of the interface is marked by blue solid line. Area I and II are separated by blue dashed line. Radial Wiener Filter within Gatan Digital Micrograph is used to reduce noise in the Zcontrast images.

(a)

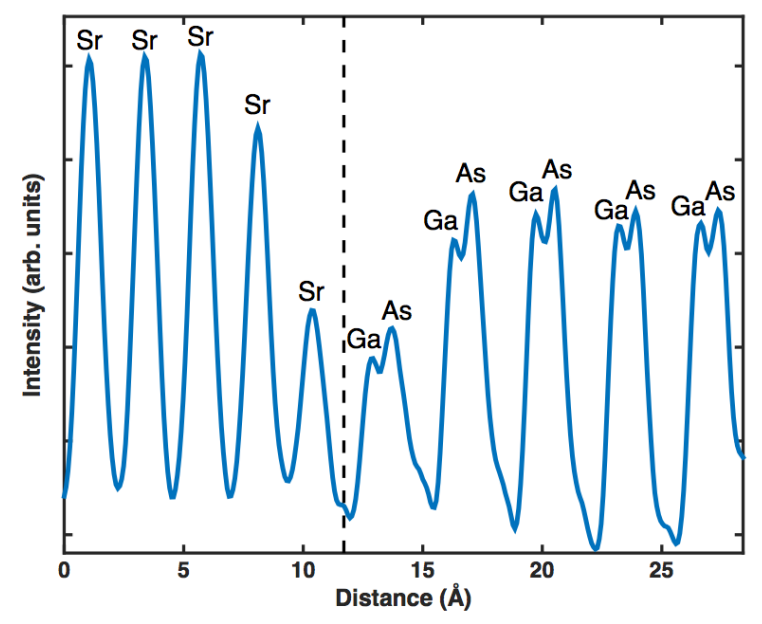

(b)

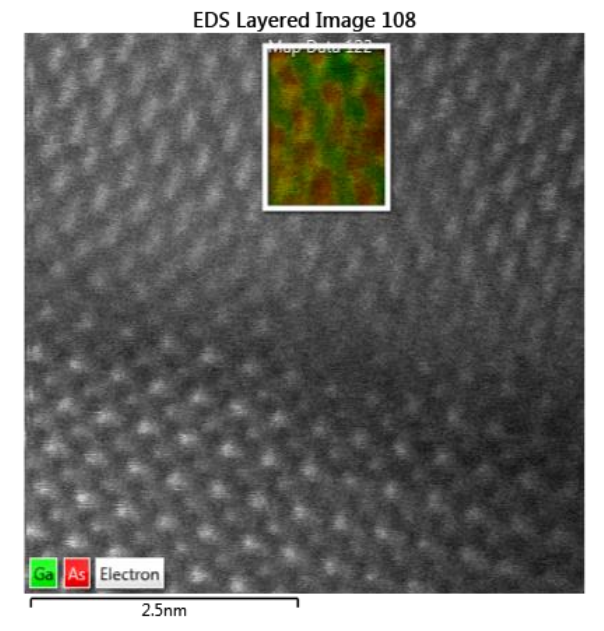

Figure 2 (a) Intensity scan of the $\mathrm{Sr} / \mathrm{GaAs}$ atomic column in the Z-contrast image in Figure 1b. The dashed line denotes the interface. The intensity is averaged for the eight $\mathrm{Sr} / \mathrm{GaAs}$ atomic columns in Area $\mathrm{I}$ and is smoothed using Gaussian function. Since the intensity is proportional to $\mathrm{Z}^{2}$, where $\mathrm{Z}$ is the atomic number, the intensity of $\mathrm{Ga}$ atoms is slightly lower than that of As atoms. (b) (Color online) Atomic-resolution EDS mapping of GaAs near the interface. Ga (As) atoms locate at the lower (upper) positions in the dumbbells. 